\title{
Article \\ Evaluation of Physical Properties of Zirconia Suspension with Added Silane Coupling Agent for Additive Manufacturing Processes
}

\author{
Jae-Gon Jang ${ }^{1,+}$, Jin-Ho Kang ${ }^{1,+} \mathbb{D}$, Kwang-Bum Joe ${ }^{2}$, Kumaresan Sakthiabirami ${ }^{1,3}$, Kyoung-Jun Jang ${ }^{1}$, \\ Mee-Jin Jun ${ }^{4}$, Gye-Jeong $\mathrm{Oh}^{3}$, Chan Park ${ }^{1}$ (D) and Sang-Won Park ${ }^{1,3, *}$
}

check for

updates

Citation: Jang, J.-G.; Kang, J.-H.; Joe, K.-B.; Sakthiabirami, K.; Jang, K.-J.; Jun, M.-J.; Oh, G.-J.; Park, C.; Park, S.-W. Evaluation of Physical Properties of Zirconia Suspension with Added Silane Coupling Agent for Additive Manufacturing Processes. Materials 2022, 15, 1337. https://doi.org/10.3390/ma15041337 Academic Editors: Rodrigo Moreno and Matthias Kern

Received: 24 December 2021

Accepted: 9 February 2022

Published: 11 February 2022

Publisher's Note: MDPI stays neutral with regard to jurisdictional claims in published maps and institutional affiliations.

Copyright: (C) 2022 by the authors. Licensee MDPI, Basel, Switzerland. This article is an open access article distributed under the terms and conditions of the Creative Commons Attribution (CC BY) license (https:// creativecommons.org/licenses/by/ $4.0 /)$.
1 Department of Prosthodontics, School of Dentistry, Chonnam National University, Gwangju 61186, Korea; jangjaegon@naver.com (J.-G.J.); jhk.bme1002@gmail.com (J.-H.K.); sakthikarthi.dentist@gmail.com (K.S.); zircong@naver.com (K.-J.J.); upgradepc@hanmail.net (C.P.)

2 School of Dentistry, Chonnam National University, Gwangju 61186, Korea; poopoopooy@naver.com

3 Biomedical Evaluation and Research Centre, School of Dentistry, Chonnam National University, Gwangju 61186, Korea; timer22@naver.com

4 Department of Dental Hygiene, Gwangju Health University, Gwangju 62287, Korea; jmejin@hanmail.net

* Correspondence: psw320@chonnam.ac.kr; Tel.: +82-62-530-5842

+ These authors contributed equally to this work.

\begin{abstract}
In this study, we have analysed the effects of a silane coupling agent on the volume fraction of zirconia for digital light processing (DLP)-based additive manufacturing processes. Zirconia suspension was prepared by the incorporation of silane-modified zirconia particles (experimental group) or untreated zirconia particles (control group). Furthermore, the control and experimental group were subdivided into three groups based on the volume fraction $(52,54$, and $56 \mathrm{vol} \%$ ) of zirconia particles. The disk-shaped zirconia samples were 3D (three-dimensional) printed using the DLP technique and their physical and mechanical properties were evaluated. The addition of a silane coupling agent to the zirconia samples was found to have influence of about $6 \%$ on the hardness and biaxial flexural strength. Moreover, the decrease in minute air gaps inside the zirconia layers significantly increased the material density (visualized from the microstructure analysis). Thus, from this study, it was established that the silane-modified zirconia particles had a positive effect on the physical properties of the zirconia parts.
\end{abstract}

Keywords: additive manufacturing; digital light processing (DLP) technology; zirconia suspension; silane coupling agent; physical property

\section{Introduction}

Various materials have been used for prostheses in dental clinics, such as titanium, cobalt-chromium, zirconia, and glass ceramics [1-7]. Among them, ceramic has been widely used and actively investigated, especially with the development of computeraided design/computer-aided manufacturing (CAD/CAM) technology. Thus far, dental ceramics that have been widely utilised in clinics include dental Empress and InCeram Alumina. However, these materials do not satisfy the physical properties, such as strength, required by dental clinics [1-3]. To improve the properties of these ceramics, research into zirconia is ongoing. Several studies on zirconia have found that this compound enhances material fracture strength and biocompatibility; it has been widely applied as a material for dental clinic-fabricated prostheses and artificial joints [8]. Zirconia ceramic prostheses have been increasingly used because patients demand better mechanical properties and aesthetics $[9,10]$. In addition, CAD/CAM technology has been applied to clinic care for dental restorations employing zirconia. However, the use of CAD/CAM to produce zirconia prostheses may produce rough scratches or form defects on the prosthesis surface, eventually leading to cracks and fracture. Furthermore, the fabrication of highly complex 
shapes using CAD/CAM is extremely difficult [10-12]. To overcome these disadvantages, research on additive manufacturing and formulations for additive manufacturing is actively being conducted. Additive manufacturing is a method that involves stacking the layers of materials using digital image processing techniques [13]. Recent research indicates that dental prostheses manufactured through additive manufacturing present acceptable density compared with those produced by milling or traditional methods [14-18].

Additive manufacturing includes spot scanning-based stereolithography, which uses a stereolithography apparatus (SLA), digital light processing (DLP), and Polyjet printing based on mask image projection [7]. Of these, SLA and DLP are technologies that print in layers using a photocuring reaction of photosensitive resins. The DLP technology stacks and hardens a formulated liquid photocurable resin through a photocuring process involving projected light according to the type and location of the shape to be moulded $[19,20]$. The main advantages of DLP are as follows: the printed product is highly dense and has a luminous surface, and the work speed is fast because the resin is moulded in face units. In addition, DLP has a faster production speed than the SLA process and enables the low-cost fabrication of samples with a small sample size [20,21]. For these reasons, the DLP system is considered suitable for additive manufacturing printers for dental ceramic production. However, despite the advances in this technology, preventing the formation of micro-cracks on the zirconia surface is difficult. The physical properties (e.g., strength) of the product produced by additive manufacturing are found to be extremely inadequate for actual clinical application [22]. To resolve this issue, a silane coupling agent can be added to the suspension for additive manufacturing. The agent combines with the surface of inorganic fillers or glass fibres that are mixed with the polymer on one side of the molecules, but has an affinity with the matrix resin on the other side. Dispersibility is enhanced, and the interfacial adhesiveness among inorganic fillers or between the glass fibre and matrix polymer improves. An organic coating based on a silane coupling agent is applied to enhance surface characteristics [23-26]. Its use was found to improve the strength of each sample. Studies indicate that the silane coupling agent improves the zirconia surface by promoting properties, such as surface strength and abrasion as well as friction resistance [27]. More importantly, silane coupling agents reduce the number of hydroxyl groups on the surface of the modified filler, increasing hydrophobicity and improving dispersion in polymer matrices and resins. This has been found to improve the strength of samples by limiting the hydrophilicity of the filler and increasing the reactivity of the filler or compatibility with the selected polymer depending on the type of silane functional group [28-31].

Methyltrimethoxysilane (MTMS), an organosilane with one methyl group and three hydrolyzable methoxy substituents, has been successfully used to impart hydrophobicity and oleophobicity to a variety of substrates. As previously reported, MTMS controls the hydrolysis conditions, so that the hydrolysis and coating processes are usually in simple ambient aqueous conditions, which represents an obvious advantage and is easily compatible with existing manufacturing processes [32].

In this study, the zirconia suspensions with a volume fraction of $52-56 \%$ without added silane were set as the control group [33], whereas those with the coupling agent were set as the experimental groups. The groups were prepared to compare the physical properties of zirconia specimens after sintering based on the incorporation or absence of a silane coupling agent. The null hypothesis of this study is that there is no difference in the physical and mechanical properties of the 3D printed zirconia samples with and without the addition of a silane coupling agent according to volume fraction.

\section{Materials and Methods}

\subsection{Preparation of Zirconia Photocurable Suspension}

Commercially available zirconia powder (TZ-3Y, Tosho, Japan) with an average particle size of $310 \mathrm{~nm}$ was used. Three monomers (all obtained from Sigma Aldrich Inc., St. Louis, MO, USA), i.e., acrylic resin-based IBA (isobornyl acrylate), HDDA (1,6-hexanediol 
diacrylate), and PNPGDA (propoxylated neopentyl glycol diacrylate), were utilised to manufacture a photocurable binder. Other additives were a photoinitiator (Irgacure 819, Ciba Specialty Chemicals, Swiss), a dispersant to improve the dispersibility of zirconia powder binder (BYK-180, BYK Inc., Wesel, Germany), and a silane coupling agent (methyltrimethoxysilane coupling agent, MTMS, Duksan, Korea). MTMS mixed solution was prepared by stirring at a mass ratio of ethanol, distilled water, and MTMS (90:8:2) for $1 \mathrm{~h}$. Then, $100 \mathrm{~g}$ of zirconia was mixed with the MTMS mixed solution. After adding the particles, it was ball milled for $24 \mathrm{~h}$ and then dried overnight at room temperature. The physical properties of the silane-modified zirconia particles were evaluated using FT-IR spectroscopy (Spectrum 400, Perkin Elmer, USA). The silane-modified zirconia powders were used to prepare suspensions of zirconia volume fractions of 52, 54, and $56 \mathrm{vol} \%$. A planetary centrifugal mixer (ARV-310, Thinky Corp., Tokyo, Japan) was used to mix the suspension homogeneously. The groups were mainly divided into $Z$ (control group) and ZS (experimental group with silane coupling agent); further, it was sub-divided into three control groups and three experimental groups based on zirconia volume fraction. The zirconia suspension compositions are summarised in Table 1 . The detailed schematic illustration of the experimental group process was displayed in Figure 1.

Table 1. Experimental groups in this study.

\begin{tabular}{|c|c|c|c|c|c|c|c|c|}
\hline \multirow{2}{*}{ Group } & & \multirow{2}{*}{$\begin{array}{l}\text { Zirconia } \\
\text { Powder }\end{array}$} & \multicolumn{3}{|c|}{ Acrylic Monomers } & \multicolumn{2}{|c|}{ Additive Agents } & \multirow{2}{*}{$\begin{array}{c}\text { Total } \\
\text { wt (vol) } \%\end{array}$} \\
\hline & & & IBA & HDDA & PNPGDA & Dispersant & Silane Coupling Agent & \\
\hline \multirow{3}{*}{ Control } & $\mathrm{Z} 52$ & $\begin{array}{c}86.45 \\
(52.00)\end{array}$ & \multicolumn{3}{|c|}{$9.23(33.43)$} & $4.32(14.57)$ & 0 & 100 \\
\hline & $\mathrm{Z} 54$ & $\begin{array}{c}87.34 \\
(54.00)\end{array}$ & \multicolumn{3}{|c|}{$8.29(30.87)$} & $4.37(15.13)$ & 0 & 100 \\
\hline & Z56 & $\begin{array}{c}88.19 \\
(56.00) \\
\end{array}$ & \multicolumn{3}{|c|}{$7.41(28.32)$} & $4.41(15.68)$ & 0 & 100 \\
\hline \multirow{3}{*}{ Experimental } & ZS52 & $\begin{array}{c}86.45 \\
(52.00)\end{array}$ & \multicolumn{3}{|c|}{$8.64(30.30)$} & $3.27(11.65)$ & $1.64(6.05)$ & 100 \\
\hline & ZS54 & $\begin{array}{c}87.34 \\
(54.00)\end{array}$ & \multicolumn{3}{|c|}{$7.68(27.62)$} & $3.32(12.10)$ & $1.66(6.28)$ & 100 \\
\hline & ZS56 & $\begin{array}{c}88.19 \\
(56.00)\end{array}$ & \multicolumn{3}{|c|}{$6.74(24.94)$} & $3.38(12.54)$ & $1.69(6.52)$ & 100 \\
\hline
\end{tabular}

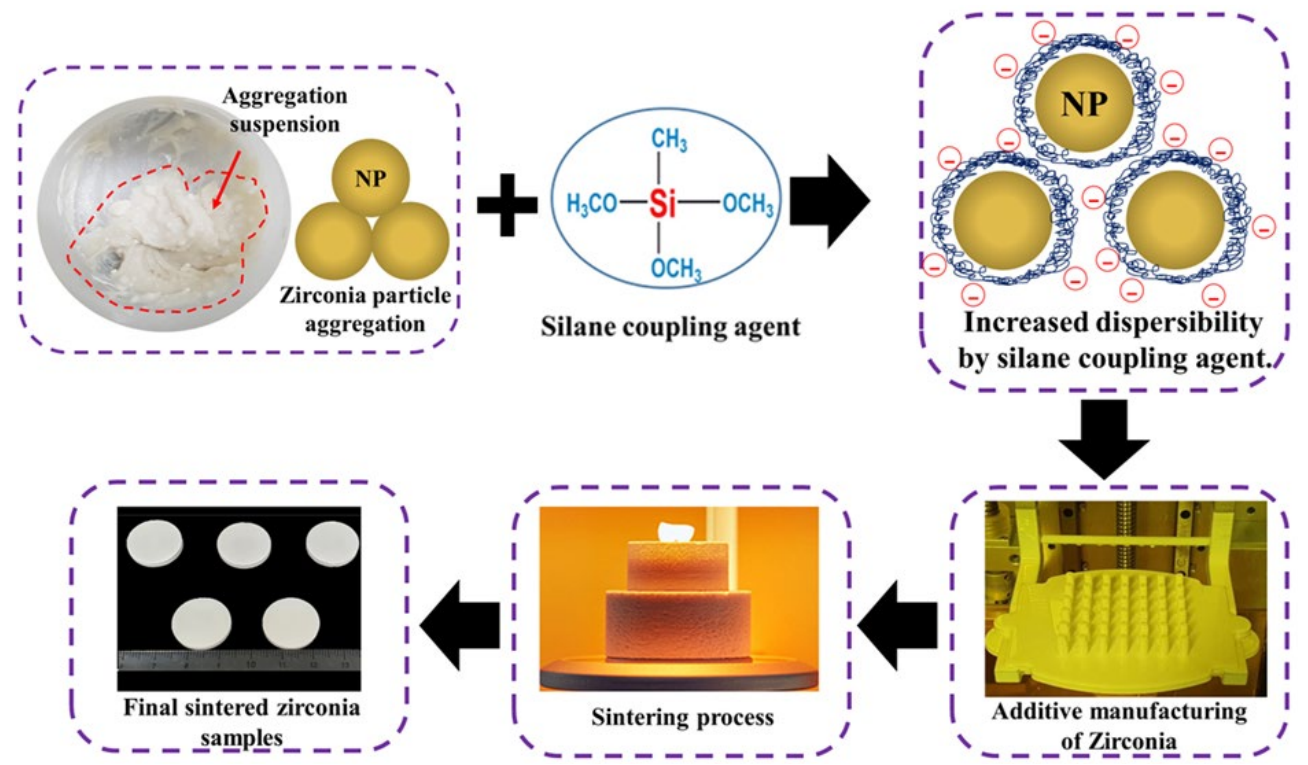

Figure 1. Schematic illustration of the experimental group process. 


\subsection{Zirconia Samples Fabrication Using Additive Manufacturing}

A DLP machine was used for light-curing the zirconia suspension (Octave Light R1, Octave Light Ltd., Shatin, Hongkong) (Figure 2); the waveband was $385 \mathrm{~nm}$. Zirconia samples were additively manufactured by setting the thickness of each layer to $50 \mu \mathrm{m}$. The STL files of samples were designed using a three-dimensional (3D) modelling program (Solid Works 2016, Dassault Systemes SOLIDWORKS Corp., Waltham, MA, USA). Each sample was fabricated in the form of a disk with diameter and height of 20 and $2 \mathrm{~mm}$, respectively.

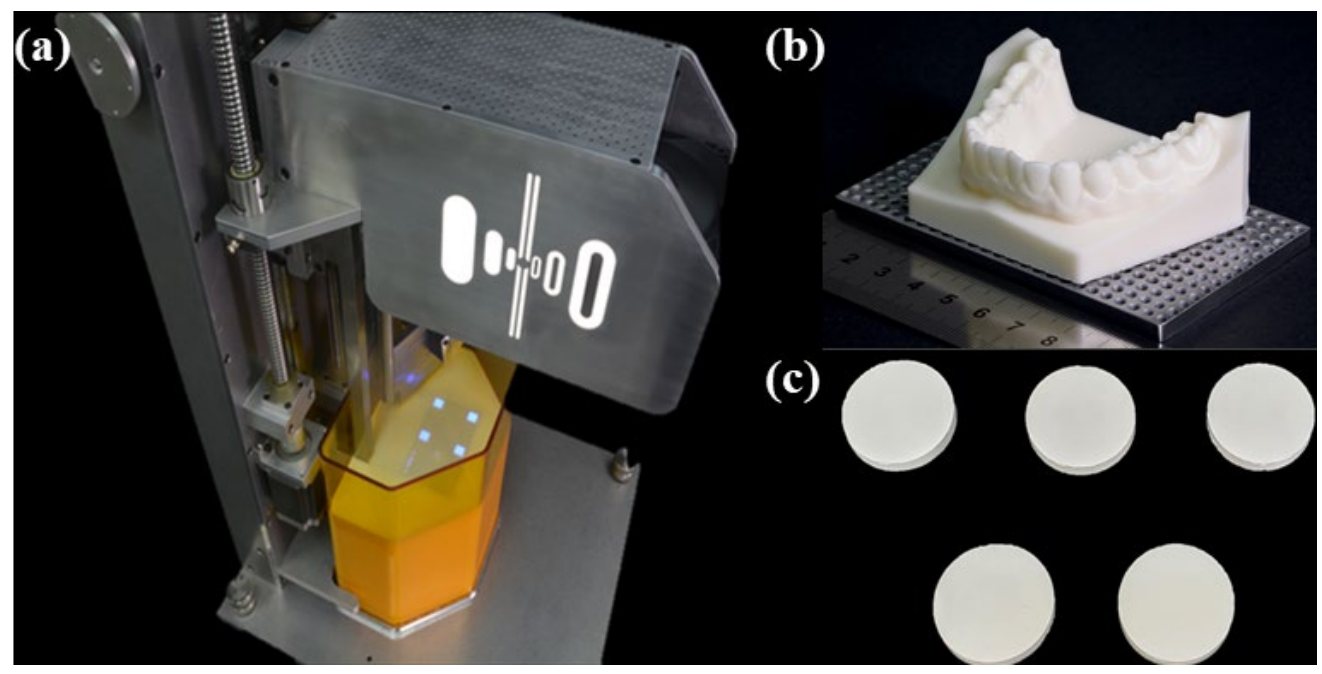

Figure 2. DLP machine used for preparing zirconia samples: (a) DLP machine curing formulations; (b) mandible and teeth model manufactured using DLP machine; (c) zirconia disk samples manufactured by DLP machine.

\subsection{Degreasing and Sintering of Zirconia Samples}

The additively manufactured zirconia samples were degreased and sintered using a sintering machine (DUOTRON PRO ex-6100, Zirconia Sintering Furnace, ADDIN Co., LTD, Suwon, Korea). The degreasing of each experimental group proceeded as follows. The temperature was gradually increased by $0.5^{\circ} \mathrm{C} / \mathrm{min}$ until it reached $200^{\circ} \mathrm{C}$ and maintained for $1 \mathrm{~h}$. Then, it was increased to $300{ }^{\circ} \mathrm{C}$ at $0.5^{\circ} \mathrm{C} / \mathrm{min}$ and again held for $1 \mathrm{~h}$. Finally, the temperature was incrementally increased to $500{ }^{\circ} \mathrm{C}$ at $0.5^{\circ} \mathrm{C} / \mathrm{min}$ and maintained for $1 \mathrm{~h}$. After the degreasing process, the sintering process proceeded as follows. The temperature was gradually increased to $1450{ }^{\circ} \mathrm{C}$ at $10^{\circ} \mathrm{C} / \mathrm{min}$ and held for $2 \mathrm{~h}$. Then, the samples were naturally cooled (Figure 3) [26]. The final sintered zirconia samples were 14.5 and $2 \mathrm{~mm}$ in diameter and height, respectively.

\subsection{Linear Shrinkage}

The linear shrinkage of fabricated zirconia samples $(n=10)$ was measured using a vernier calliper (ASTM C 326: 2009). The diameter and height of samples were measured before and after sintering according to the volume fraction of zirconia. The following formula is used:

$$
\triangle \mathrm{X}=\left\{\left(\mathrm{X}_{1}-\mathrm{X}_{2}\right) / \mathrm{X}_{1}\right\} \times 100
$$

where $X_{1}$ and $X_{2}$ are the volume $\left(\mathrm{mm}^{3}\right)$ of zirconia before and after sintering, respectively.

\subsection{Relative Density Measurement}

The relative density of zirconia samples was measured according to the volume fraction with reference to ISO 18754: 2007. After grinding the surface to reduce the influence of surface roughness, relative density is calculated using the following formula:

$$
\rho=\left(\mathrm{m}_{1} / \mathrm{m}_{3}-\mathrm{m}_{2}\right) \times \rho_{(\mathrm{Liq})},
$$


where $m_{1}$ is the dry mass $(g), m_{2}$ is the underwater mass $(g), m_{3}$ is water mass $(g)$, and $\rho_{(\operatorname{Liq})}$ is the density of liquid infiltration solution (density of water at room temperature $=1.0 \mathrm{~g} / \mathrm{m}^{3}$ ); relative density $(\%)$ is calculated by (measured density/theoretical density) $\times 100$.

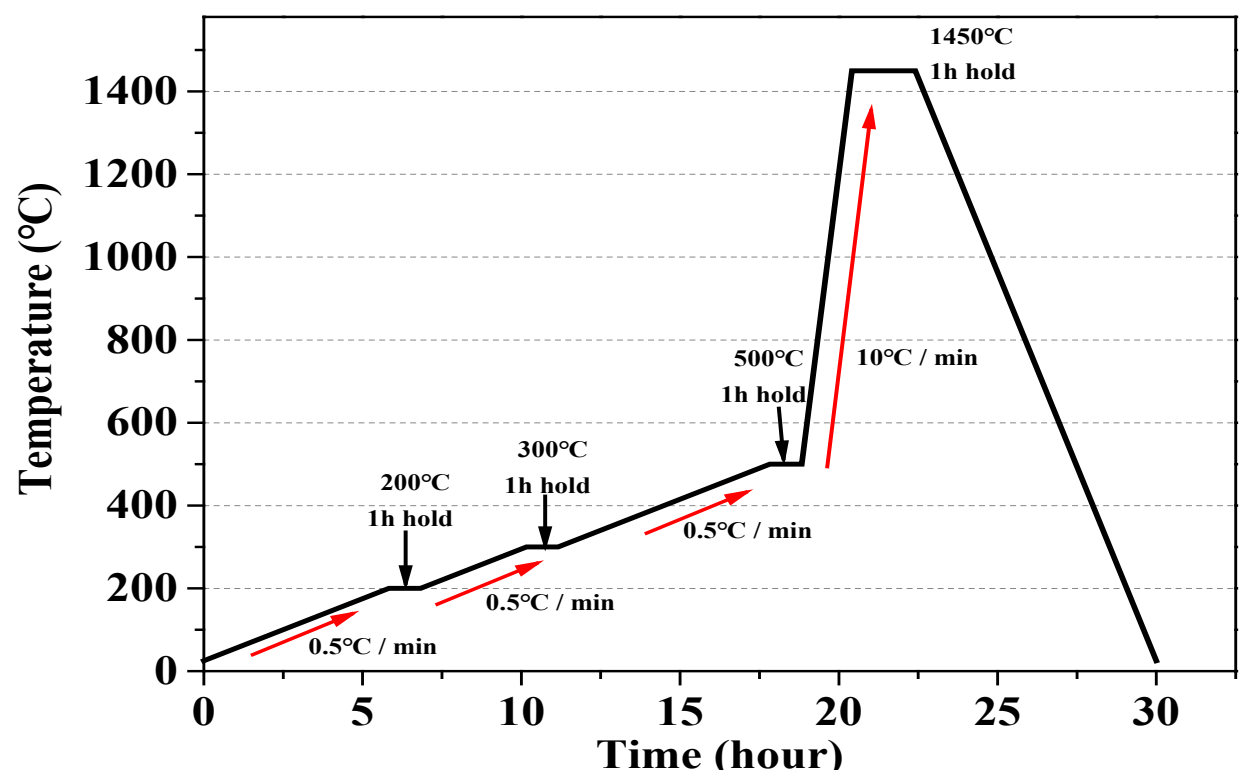

Figure 3. Sintering process of zirconia specimens (The red arrow indicates the heating rate.).

\subsection{Hardness}

To measure the hardness of the final sintered zirconia samples (each with a diameter and height of 14.5 and $2 \mathrm{~mm}$, respectively), these were fixed to multiple diamond plates and ground under constant pressure using grits in the order \#400, \#800, \#1200, and \#2400 $(n=10)$. The sample surface was finely ground using diamond pastes with $3 \mu \mathrm{m}$ and $1 \mu \mathrm{m}$ particles (MP-DS-50-0300, MTDI, Daejeon, Korea). The ground samples were pressed five times for $12 \mathrm{~s}$ with a force of $1 \mathrm{kgf}(9.8 \mathrm{~N})$ using a Micro-Vickers hardness tester (NOVA $^{\circledR} 130 / 240$, INNOVATEST, Maastricht, Nederland) to determine the micro-hardness at each instance.

\subsection{Biaxial Flexural Strength}

The flexural strength of zirconia samples pertains to the bending stress that the samples can withstand when pressure is applied to them. The biaxial flexural strength of samples depending on the volume fraction was measured according to ISO 6872. Ten samples from each experimental group were measured by loading in a universal testing machine (RB Model 301 Unitech MTM R\&B, Seongnam, Korea). The load was increased at a crosshead speed of $1 \mathrm{~mm} / \mathrm{min}$ until the samples were completely fractured. Calculations are performed using the following formulae:

$$
\begin{gathered}
\sigma=-0.2387 \mathrm{P}(\mathrm{X}-\mathrm{Y}) / \mathrm{b} 2, \\
\mathrm{X}=(1+\mathrm{v}) \ln (\mathrm{r} 2 / \mathrm{r} 3) 2+[(1-\mathrm{v}) / 2](\mathrm{r} 2 / \mathrm{r} 3)(2), \\
\mathrm{Y}=(1+\mathrm{v})[1+\ln (\mathrm{r} 1 / \mathrm{r} 3) 2]+(1-\mathrm{v})(\mathrm{r} 1 / \mathrm{r} 3)(2),
\end{gathered}
$$

where $\sigma$ is the biaxial flexural strength $(\mathrm{MPa}) ; \mathrm{P}$ is the maximum load at fracture (Newtons); $\mathrm{b}$ is the sample thickness $(\mathrm{mm})$; $\mathrm{v}$ is Poisson's Ratio $(\mathrm{v}=0.25)$; $\mathrm{r} 1$ is the radius of the circle supporting the load (mm); r2 is the radius of the circle to which the load is applied (mm); and $\mathrm{r} 3$ is the sample radius ( $\mathrm{mm})$. 


\subsection{Evaluation of Microstructure of Final Sintered Zirconia Samples}

To observe the microstructure of zirconia samples, their surfaces were ground using diamond pastes with $3 \mu \mathrm{m}$ and $1 \mu \mathrm{m}$ particles (MP-DS-50-0300, MTDI, Daejeon, Korea). Then, they were cleaned with alcohol and distilled water in an ultrasonic cleaner (JAC-2010, KODO Technical Research Co., Hwaseong, Korea) for 20 min. To observe the cross-section, the samples were cleaned with alcohol and distilled water for $20 \mathrm{~min}$. The platinum coating was applied to sample surfaces using a sputter coater (108 Auto, Cressington Scientific Instruments, Ltd., Oxhey, UK). After coating, the zirconia sample layers were observed using a scanning electron microscope (FE-SEM, JEOL, JSM-7500F, Tokyo, Japan).

\subsection{Statistical Analysis}

The sample size was evaluated using G-Power 3.1 software (University of Dusseldorf, Dusseldorf, Germany) [5]. The experimental results were statistically processed by one-way analysis of variance using the SPSS software package (SPSS Version 20.0, SPSS Inc., Chicago, IL, USA), and post tested with the Tukey HSD test. All results were tested for statistical significance with $p<0.05$.

\section{Results}

\subsection{FT-IR Analysis}

FT-IR spectroscopy was performed to determine the organic characterization, i.e., the effect of silanization of zirconia particles. As shown in Figure 4, the stretching vibration of $-\mathrm{CH}$ appears at $2983 \mathrm{~cm}^{-1}$ and $2900 \mathrm{~cm}^{-1}$, respectively. The bending vibration of $-\mathrm{CH}$ is $1405 \mathrm{~cm}^{-1}$. At $1270 \mathrm{~cm}^{-1}$ the stretching vibration of $\mathrm{C}-\mathrm{O}$ appears, while the stretching vibration of $-\mathrm{Si}-\mathrm{O}-$ attached to the $-\mathrm{CH}$ is at $1052 \mathrm{~cm}^{-1}$, the stretching vibration of $-\mathrm{O}-\mathrm{O}-$ appears at $890 \mathrm{~cm}^{-1}$. From the results, it was confirmed that the zirconia particles were treated with the silane coupling agent, and a zirconia slurry was prepared using the treated powder.

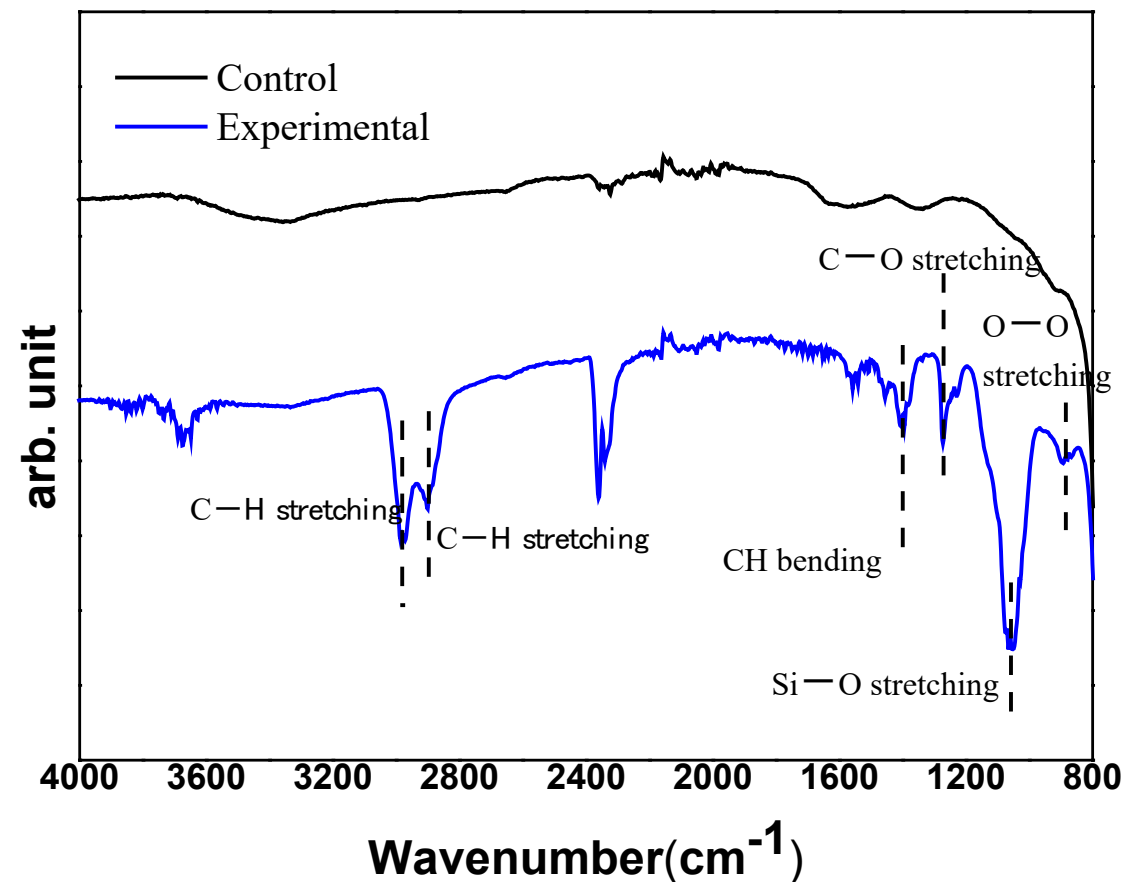

Figure 4. FT-IR spectra of the zirconia powder before and after silanization.

\subsection{Linear Shrinkage}

The linear shrinkage of additively manufactured zirconia samples was measured according to the zirconia volume fraction by comparing them before and after sintering (Table 2 and Figure 5). 
Table 2. Average linear shrinkage of printed zirconia sample.

\begin{tabular}{ccc}
\hline \multirow{2}{*}{ Groups } & \multicolumn{2}{c}{ Linear Shrinkage (\%) } \\
\cline { 2 - 3 } & Control & Experimental \\
\hline Z52 & $19.20 \pm 0.31$ & $19.14 \pm 0.17$ \\
Z54 & $19.31 \pm 0.23$ & $19.23 \pm 0.20$ \\
& $19.15 \pm 0.29$ & $19.21 \pm 0.09$ \\
\hline
\end{tabular}

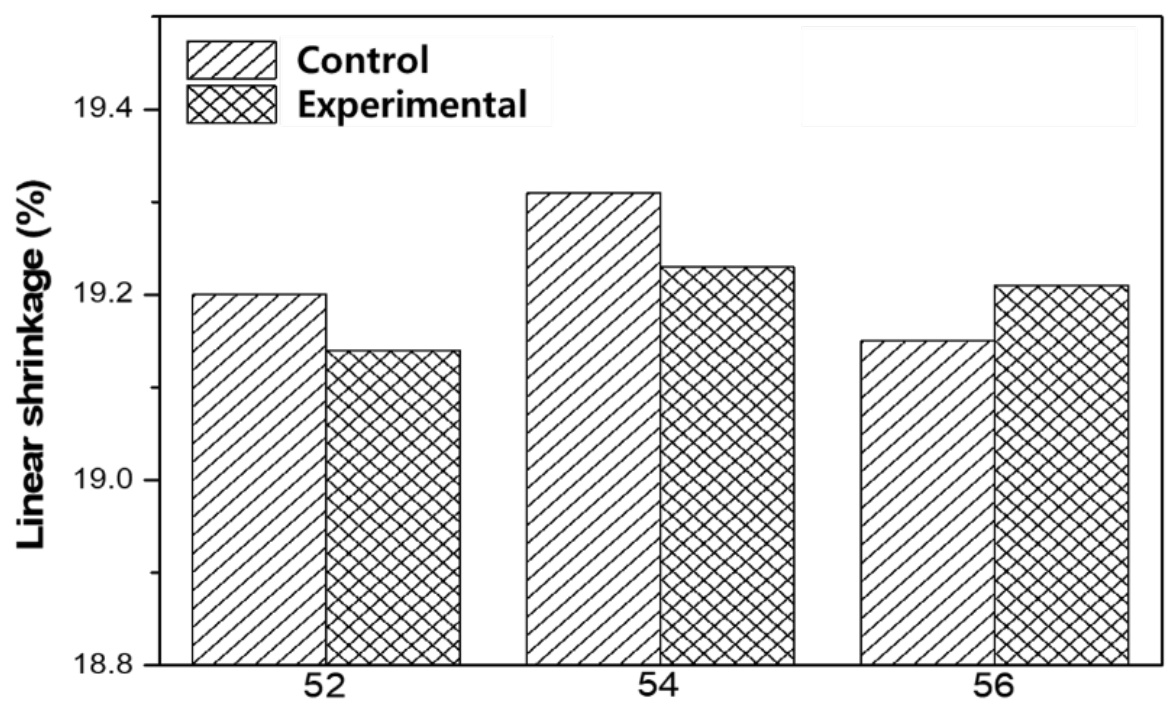

Figure 5. Comparison of linear shrinkage between control and experimental groups after final sintering.

In the previous experiment, the shrinkage range of zirconia powder samples sintered at $1200-1400{ }^{\circ} \mathrm{C}$ was $19-20 \%$; consequently, the average linear shrinkage of zirconia ceramic was found to be $18-25 \%$ [11]. The linear contraction rate of experimental and control groups exhibited a constant pattern of approximately $18-20 \%$; however, no significant difference in the contraction rate between the two groups was observed. Moreover, it should be noted that in all groups, linear shrinkage was greater in height than in diameter. This is attributed to the occurrence of linear shrinkage in sample height during sintering, indicating that the action of gravitational force on height exceeds the effect of this force on diameter. Moreover, the greater linear shrinkage in height than in diameter was presumed to occur because the evaporation of resin in each layer at high temperatures caused the shrinkage of air gaps [34,35].

\subsection{Relative Density}

The relative density values of additively manufactured zirconia samples in control groups and experimental groups are shown in Table 3 and Figure 6.

Table 3. Relative density of printed zirconia; different letters represent significant differences $(p<0.05)$.

\begin{tabular}{ccc}
\hline \multirow{2}{*}{ Groups } & \multicolumn{2}{c}{ Relative Density (\%) } \\
\cline { 2 - 3 } & Control & Experimental \\
\hline Z52 & $94.89 \pm 0.35^{\mathrm{a}}$ & $19.14 \pm 0.09^{\mathrm{a}}$ \\
Z54 & $95.65 \pm 0.67^{\mathrm{b}}$ & $19.06 \pm 0.10^{\mathrm{b}}$ \\
\hline
\end{tabular}




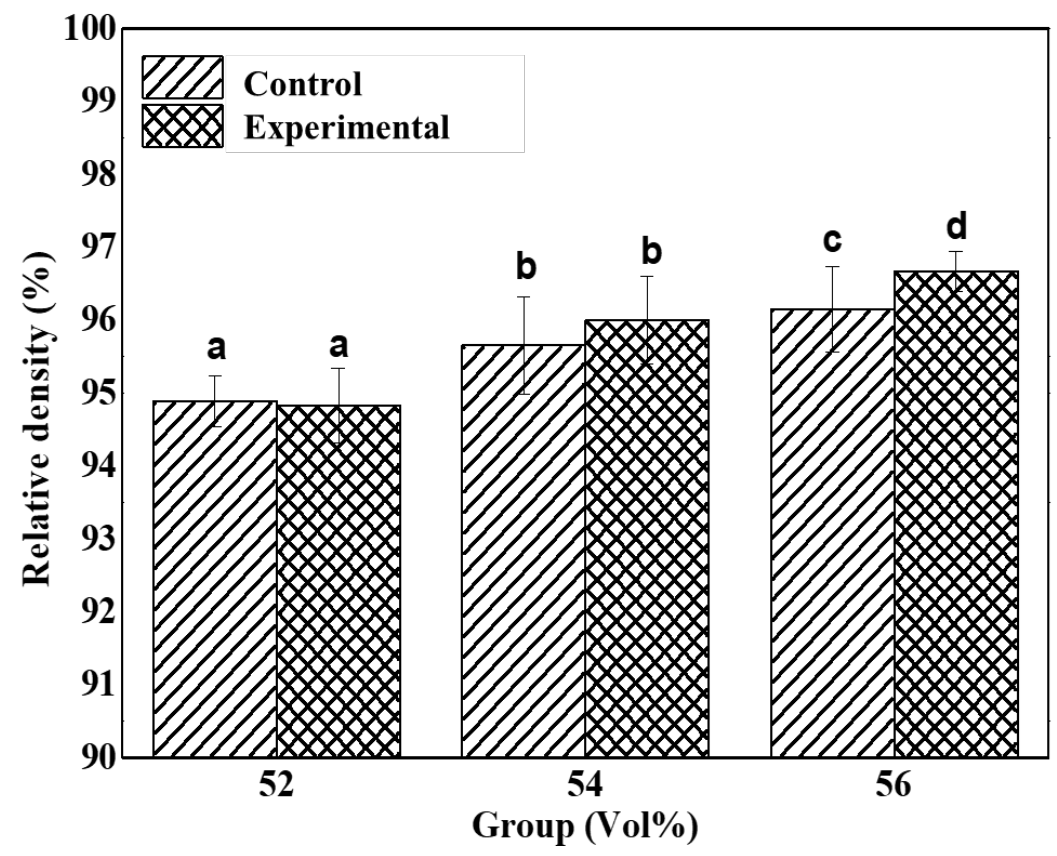

Figure 6. Comparison of relative density between control and experimental groups; different letters represent significant differences $(p<0.05)$.

The difference between Z52, Z54, and Z56 was significant $(p<0.05)$. As for the experimental groups, the difference between ZS54 and ZS56 was insignificant, whereas that between these two and ZS52 was significant. Based on the addition of silane coupling agent, the difference between Z52 and ZS52 and that between Z54 and ZS54 did not significantly differ; however, a significant difference between Z56 and ZS56 ( $p>0.05)$ was observed. Regardless of whether the silane coupling agent was added to the samples, the relative density increased as the volume fraction of zirconia increased. However, when a high-volume fraction of zirconia was added, density was further increased with the addition of a silane coupling agent. The silane coupling agent was presumed to enable the homogeneous dispersion of zirconia particles in the suspension. As a result, density was evenly distributed during particle growth [36]. The higher the relative density, the higher the sample density; hence, mechanical properties, such as strength, can be enhanced.

\subsection{Hardness Measurement}

The Vickers hardness evaluation results of control and experimental groups were shown in Table 4 and Figure 7.

Table 4. Vickers hardness of printed zirconia; different letters represent significant differences $(p<0.05)$.

\begin{tabular}{ccc}
\hline \multirow{2}{*}{ Groups } & \multicolumn{2}{c}{ Vickers hardness (HV) } \\
\cline { 2 - 3 } & Control & Experimental \\
\hline Z52 & $1573 \pm 21.31^{\mathrm{a}}$ & $1666 \pm 14.61^{\mathrm{d}}$ \\
$\mathrm{Z} 54$ & $1648 \pm 48.17^{\mathrm{b}}$ & $1748 \pm 20.33^{\mathrm{e}}$ \\
$\mathrm{Z} 56$ & $1777 \pm 22.19^{\mathrm{c}}$ & $1848 \pm 24.04^{\mathrm{f}}$ \\
\hline
\end{tabular}




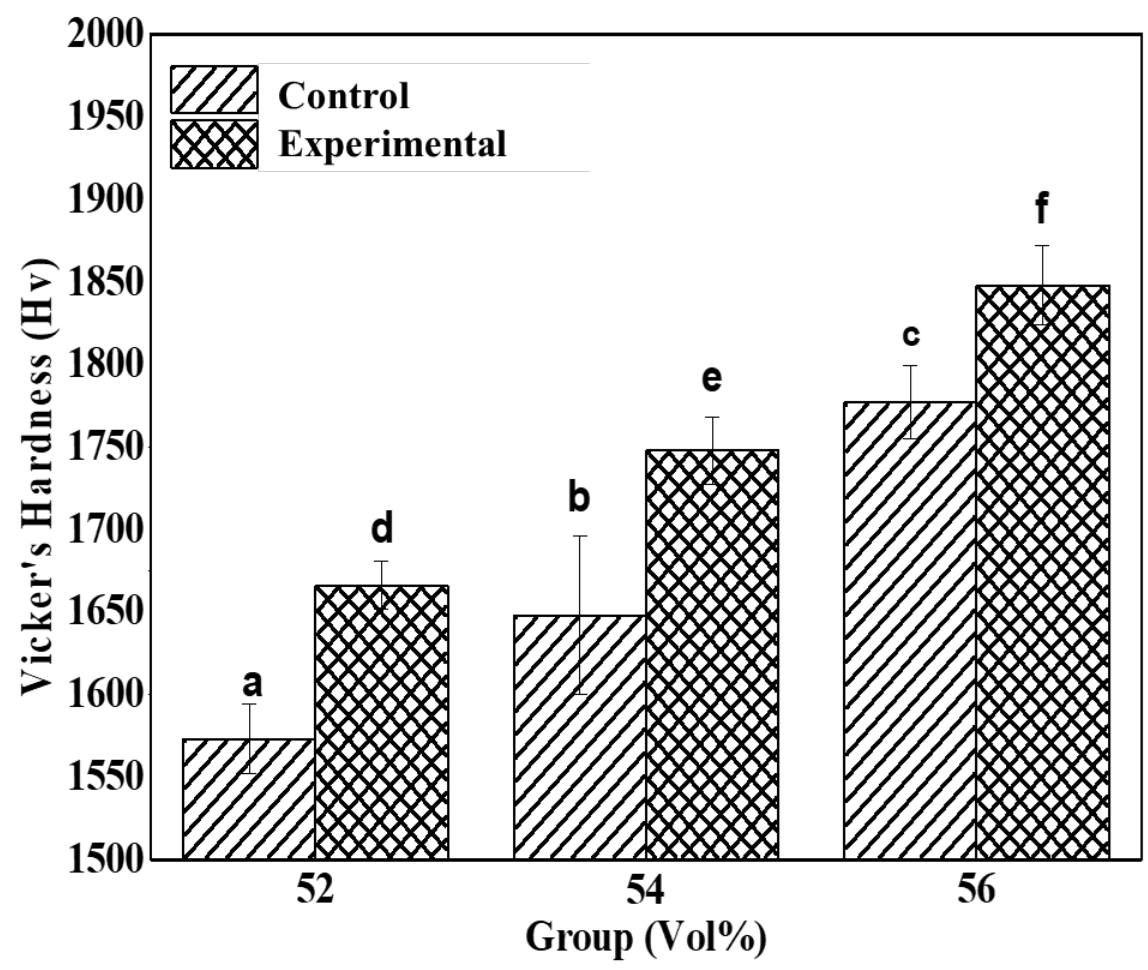

Figure 7. Comparison of Vickers hardness between control and experimental groups; different letters represent significant differences $(p<0.05)$.

All experimental groups significantly differed in average hardness $(p<0.05)$. All zirconia samples exhibited significant variations in Vickers average hardness $(p<0.05)$. Group ZS56, which had a zirconia volume fraction of $56 \mathrm{vol} \%$ and contained a silane coupling agent, exhibited the highest hardness value; it was followed by Z56, ZS54, ZS52, Z54, and Z52. The hardness values among these groups significantly differed (Figure 5). The addition of silane coupling agents to the samples promoted the homogeneous commixture of organic (photocurable polymers) and inorganic (zirconia) substances. As a result, dispersibility improved and hardness increased $[21,28,36]$.

\subsection{Biaxial Flexural Strength}

From the biaxial flexural strength measurement of samples, the average biaxial flexural strength was displayed in Table 5 and Figure 8.

Table 5. Vickers hardness of printed zirconia; different letters represent significant differences $(p<0.05)$.

\begin{tabular}{ccc}
\hline \multirow{2}{*}{ Group } & \multicolumn{2}{c}{ Flexural Strength (MPa) } \\
\cline { 2 - 3 } & Control & Experimental \\
\hline Z52 & $346 \pm 6.96^{\mathrm{a}}$ & $368 \pm 8.62^{\mathrm{d}}$ \\
Z54 & $384 \pm 3.68^{\mathrm{b}}$ & $407 \pm 8.87^{\mathrm{e}}$ \\
\hline
\end{tabular}




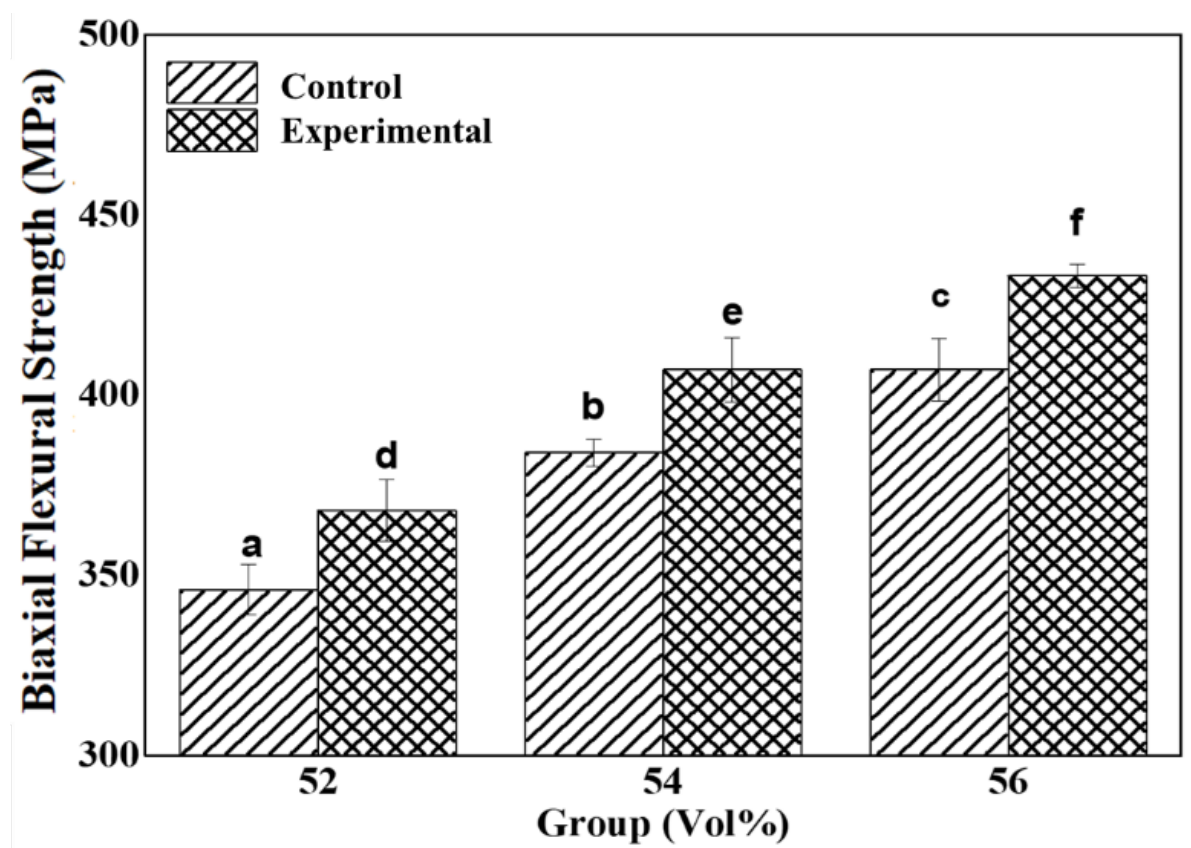

Figure 8. Comparison of biaxial flexural strength between control and experimental groups; different letters represent significant differences $(p<0.05)$.

The biaxial flexural strengths between Z52 and ZS52, between Z54 and ZS54, and between Z56 and ZS56 significantly differed according to the added silane coupling agent $(p<0.05)$. Similar to the results of Vickers hardness measurement, the following results are attributed to the commixture of photocuring polymer and zirconia promoted by the addition of a silane coupling agent, thereby increasing dispersibility [37]. Accordingly, the biaxial flexural strength of groups with the silane coupling agent increased.

\subsection{Microstructure Evaluation}

The fractured sections of additively manufactured zirconia samples were observed using the scanning electron microscope for microstructure evaluation according to the zirconia volume fraction (Figure 9). The layers of additively manufactured zirconia samples were examined through this observation. The smaller the zirconia volume fraction, the more air gaps were observed (red arrows in Figure 9). The variations in relative density measurements were confirmed to be caused by these air gaps.

These observations are considered to support the measurements of low density and strength because of the occurrence of numerous air gaps in groups with no added silane coupling agent. In addition, the air gaps in groups with the added silane coupling agent were smaller in size compared with those in groups with no added coupling agent.

The images of the layers of additively manufactured zirconia samples were captured through scanning electron microscopy. The layers were observed to be smaller and more constant in groups with the added silane coupling agent compared with those without the agent. Moreover, the proportion of air gaps caused by the debinding of zirconia samples during sintering was found to decrease and then remain constant. If the air gaps were not constant, micro-cracks were more likely to occur due to external forces that may degrade strength, hardness, and physical properties [33,38]. If zirconia-based prostheses are applied in actual dental clinical practice, the foregoing can increase the possibility of prosthesis breakage caused by the masticatory force of patients.

Therefore, considering that the air gaps are reduced and remain constant, improved strength can be expected from additively manufactured zirconia with the added silane coupling agent.

This study demonstrated the effect of silane on the volume fraction of zirconia. However, future experiments are needed to evaluate the dispersion of the silane-modified 
zirconia particles in the suspension by varying experimental parameters associated with the salinisation of zirconia particles, such as $\mathrm{pH}$, solution composition, and hydrolysis time.
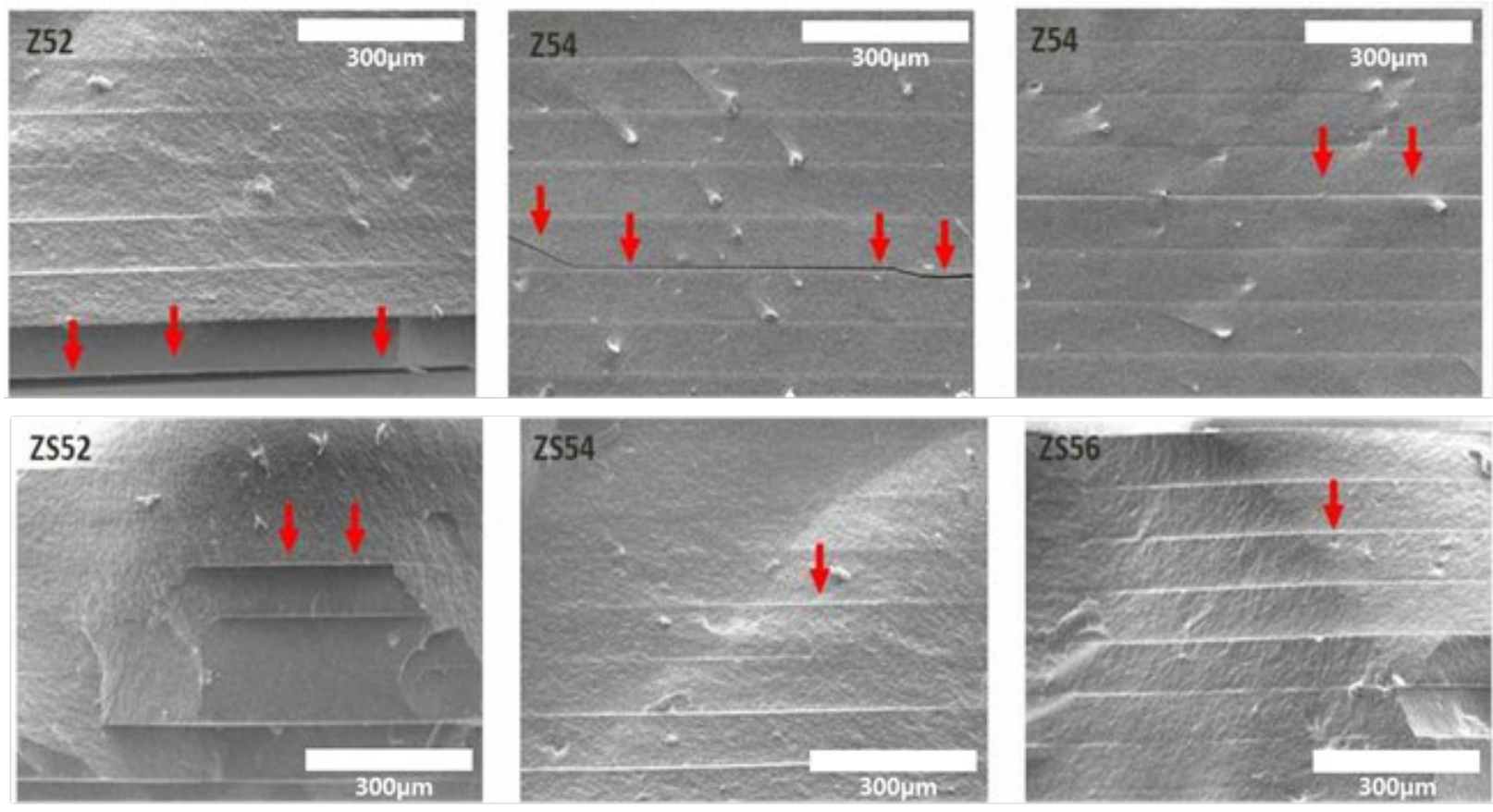

Figure 9. FE-SEM images of microstructure and layer-by-layer observation of the final sintered zirconia specimen (Red arrows indicate microcracks and pores).

\section{Conclusions}

In this study, the preparation of zirconia suspensions with the addition of a silane coupling agent (approximately $2 \%$ of zirconia volume fraction) for DLP additive manufacturing technology has been demonstrated. It was established that the density of the printed zirconia samples significantly increased with an increase in the zirconia volume fraction $(52,54$, and $56 \mathrm{vol} \%)$. Interestingly, the experimental group (ZS56) with the addition of silane coupling agent to the suspension of $56 \mathrm{vol} \%$ exhibits improved strength and hardness (5-6\%) compared to those without silane coupling agent (control group). However, it is essential to focus future research activities on enhancing the dispersibility of zirconia suspension using the silane coupling agent for DLP printing. In future experiments, we plan to evaluate the dispersion stability of the zirconia suspension by varying conditions, such as $\mathrm{pH}$ control, solution composition, and hydrolysis time, for the encapsulation of the zirconia particles with the silane coupling agent.

Author Contributions: Conceptualization and writing-original draft preparation, S.-W.P., J.-G.J. and J.-H.K.; writing-review and editing, S.-W.P., J.-H.K., K.S. and C.P.; Methodology, K.-B.J. and J.-G.J.; visualization, K.-J.J. and M.-J.J.; supervision and funding acquisition, G.-J.O. and S.-W.P. All authors have read and agreed to the published version of the manuscript.

Funding: This work was supported by a National Research Foundation of Korea Grant funded by the Korean government (MSIP) [Grant no. 2019R1A2C108945613 and 2020R111A1A01073015].

Institutional Review Board Statement: Not applicable.

Informed Consent Statement: Not applicable.

Data Availability Statement: All the data have been illustrated in the manuscript.

Conflicts of Interest: The authors declare no conflict of interest. 


\section{References}

1. Mclean, J.W. The reinforcement of dental porcelain with ceramic oxides. Br. Dent. J. 1965, 119, 251-267. [PubMed]

2. Shareef, M.; Van Noort, R.; Messer, P.; Piddock, V. The effect of microstructural features on the biaxial flexural strength of leucite reinforced glass-ceramics. J. Mater. Sci. Mater. Med. 1994, 5, 113-118. [CrossRef]

3. Jones, D.W. Development of dental ceramics. An historical perspective. Dent. Clin. North Am. 1985, 29, 621-644. [PubMed]

4. Ammarullah, M.I.; Afif, I.Y.; Maula, M.I.; Winarni, T.I.; Tauviqirrahman, M.; Akbar, I.; Basri, H.; van der Heide, E.; Jamari, J. Tresca Stress Simulation of Metal-on-Metal Total Hip Arthroplasty during Normal Walking Activity. Materials 2021, $14,7554$. [CrossRef] [PubMed]

5. Ramaraj, S.; Kim, M.-A.; Rosa, V.; Prasanna, N.; Shon, W.-J.; Min, K.-S. Combined Effect of Melittin and DNase on Enterococcus faecalis Biofilms and Its Susceptibility to Sodium Hypochlorite. Materials 2020, 13, 3740. [CrossRef] [PubMed]

6. Sakthiabirami, K.; Kang, J.H.; Jang, J.G.; Lim, H.P.; Yun, K.D.; Park, C.; Yim, E.K.; Park, S.W.; Ko, K.T.; Oh, G.J. Effect of Argon Plasma Treatment on Surface-Treated Titanium. Adv. Dent. Oral Health 2019, 11, 158-164.

7. Kumaresan, S.; Vaiyapuri, S.; Kang, J.-H.; Dubey, N.; Manivasagam, G.; Yun, K.-D.; Park, S.-W. Additive Manufactured Zirconia-Based Bio-Ceramics for Biomedical Applications [Online First], IntechOpen. 19 January 2022. Available online: https: / / www.intechopen.com/online-first/80125 (accessed on 10 February 2022). [CrossRef]

8. Sakthiabirami, K.; Kim, J.W.; Kang, J.H.; Jang, K.J.; Oh, G.J.; Fisher, J.G.; Yun, K.D.; Lim, H.P.; Park, S.W. Tailoring interfacial interaction through glass fusion in glass/zinc-hydroxyapatite composite coatings on glass-infiltrated zirconia. Ceram. Int. 2018, 44, 16181-16190. [CrossRef]

9. Schriwer, C.; Skjold, A.; Gjerdet, N.R.; Øilo, M. Monolithic zirconia dental crowns. Internal fit, margin quality, fracture mode and load at fracture. Dent. Mater. 2017, 33, 1012-1020. [CrossRef]

10. D'Addazio, G.; Santilli, M.; Rollo, M.L.; Cardelli, P.; Rexhepi, I.; Murmura, G.; Al-Haj Husain, N.; Sinjari, B.; Traini, T.; Özcan, M Fracture resistance of zirconia-reinforced lithium silicate ceramic crowns cemented with conventional or adhe-sive systems: An in vitro study. Materials 2020, 13, 2012. [CrossRef]

11. Denry, I.; Kelly, J.R. State of the art of zirconia for dental applications. Dent. Mater. 2008, 24, 299-307. [CrossRef]

12. Preis, V.; Behr, M.; Hahnel, S.; Handel, G.; Rosentritt, M. In vitro failure and fracture resistance of veneered and full-contour zirconia restorations. J. Dent. 2012, 40, 921-928. [CrossRef] [PubMed]

13. Preis, V.; Behr, M.; Handel, G.; Schneider-Feyrer, S.; Hahnel, S.; Rosentritt, M. Wear performance of dental ceramics after grinding and polishing treatments. J. Mech. Behav. Biomed. Mater. 2012, 10, 13-22. [CrossRef] [PubMed]

14. Huang, Z.; Zhang, L.; Zhu, J.; Zhao, Y.; Zhang, X. Clinical marginal and internal fit of crowns fabricated using different CAD/CAM technologies. J. Prosthodont. 2015, 24, 291-295. [CrossRef] [PubMed]

15. Dawood, A.; Marti, B.M.; Sauret-Jackson, V.; Darwood, A. 3D printing in dentistry. Br. Dent. J. 2015, 219, 521-529. [CrossRef]

16. Park, J.-M.; Ahn, J.-S.; Cha, H.-S.; Lee, J.-H. Wear resistance of 3D printing resin material opposing zirconia and metal antagonists. Materials 2018, 11, 1043. [CrossRef] [PubMed]

17. Zeltser, C.; Lewinstein, I.; Grajower, R. Fit of crown wax patterns after removal from the die. J. Prosthet. Dent. 1985, 53, 344-346. [CrossRef]

18. Abduo, J.; Lyons, K.; Swain, M. Fit of zirconia fixed partial denture: A systematic review. J. Oral Rehabil. 2010, 37, 866-876. [CrossRef]

19. Harish, V.; Mohamed Ali, S.; Jagadesan, N.; Mohamed Ifthikar, S.S.; Debasish Basak, F.H. Evaluation of internal and marginal fit of two metal ceramic system-In vitro study. J. Clin. Diagn. Res. JCDR 2014, 8, ZC53.

20. He, R.; Liu, W.; Wu, Z.; An, D.; Huang, M.; Wu, H.; Jiang, Q.; Ji, X.; Wu, S.; Xie, Z. Fabrication of complex-shaped zirconia ceramic parts via a DLP-stereolithography-based 3D printing method. Ceram. Int. 2018, 44, 3412-3416. [CrossRef]

21. Kang, J.-H.; Jang, K.-J.; Sakthiabirami, K.; Oh, G.-J.; Jang, J.-G.; Park, C.; Lim, H.-P.; Yun, K.-D.; Park, S.-W. Mechanical properties and optical evaluation of scaffolds produced from 45S5 bioactive glass suspensions via stereolithography. Ceram. Int. 2020, 46, 2481-2488. [CrossRef]

22. Sakthiabirami, K.; Soundharrajan, V.; Kang, J.-H.; Yang, Y.P.; Park, S.-W. Three-Dimensional Zirconia-Based Scaffolds for Load-Bearing Bone-Regeneration Applications: Prospects and Challenges. Materials 2021, 14, 3207. [CrossRef] [PubMed]

23. Moreno, M.B.P.; Murillo-Gómez, F.; de Goes, M.F. Physicochemical and morphological characterization of a glass ceramic treated with different ceramic primers and post-silanization protocols. Dent. Mater. 2019, 35, 1073-1081. [CrossRef] [PubMed]

24. Moreno, M.B.P.; Murillo-Gómez, F.; De Goes, M. Effect of different ceramic-primers and silanization-protocols on glass-ceramic bond strength. Dent. Mater. 2018, 34, e80-e81. [CrossRef]

25. Villa, S.; Riani, P.; Locardi, F.; Canepa, F. Functionalization of Fe3O4 NPs by silanization: Use of amine (APTES) and thiol (MPTMS) silanes and their physical characterization. Materials 2016, 9, 826. [CrossRef]

26. Jang, K.-J.; Kang, J.-H.; Fisher, J.G.; Park, S.-W. Effect of the volume fraction of zirconia suspensions on the microstructure and physical properties of products produced by additive manufacturing. Dent. Mater. 2019, 35, e97-e106. [CrossRef]

27. Lopez de Armentia, S.; Pantoja, M.; Abenojar, J.; Martinez, M.A. Development of silane-based coatings with zirconia nanoparticles combining wetting, tribological, and aesthetical properties. Coatings 2018, 8, 368. [CrossRef]

28. Barczewski, M.; Matykiewicz, D.; Szostak, M. The effect of two-step surface treatment by hydrogen peroxide and silani-zation of flax/cotton fabrics on epoxy-based laminates thermomechanical properties and structure. J. Mater. Res. Technol. 2020, 9 , 13813-13824. [CrossRef] 
29. Nakonieczny, D.S.; Antonowicz, M.; Paszenda, Z. Surface modification methods of ceramic filler in ceramic-carbon fibre composites for bioengineering applications-A systematic review. Rev. Adv. Mater. Sci. 2020, 59, 586-605. [CrossRef]

30. Nakonieczny, D.S.; Kern, F.; Dufner, L.; Dubiel, A.; Antonowicz, M.; Matus, K. Effect of Calcination Temperature on the Phase Composition, Morphology, and Thermal Properties of $\mathrm{ZrO} 2$ and Al2O3 Modified with APTES (3-aminopropyltriethoxysilane). Materials 2021, 14, 6651. [CrossRef]

31. Lin, H.; Rosu, C.; Jiang, L.; Sundar, V.A.; Breedveld, V.; Hess, D.W. Nonfluorinated superhydrophobic chemical coatings on polyester fabric prepared with kinetically controlled hydrolyzed methyltrimethoxysilane. Ind. Eng. Chem. Res. 2019, 58, 15368-15378. [CrossRef]

32. Song, S.Y.; Park, M.S.; Lee, J.W.; Yun, J.S. A study on the rheological and mechanical properties of photo-curable ceramic/polymer composites with different silane coupling agents for SLA 3D printing technology. Nanomaterials 2018, 8, 93. [CrossRef] [PubMed]

33. Jang, K.-J.; Kang, J.-H.; Sakthiabirami, K.; Lim, H.-P.; Yun, K.-D.; Yim, E.-K.; Oh, G.-J.; Yang, H.-S.; Lee, K.-K.; Park, S.-W. Evaluation of cure depth and geometrical overgrowth depending on zirconia volume fraction using digital light processing. $J$. Nanosci. Nanotechnol. 2019, 19, 2154-2157. [CrossRef] [PubMed]

34. Shang, H.; Mohanram, A.; Olevsky, E.; Bordia, R.K. Evolution of anisotropy in hierarchical porous ceramics during sinter-forging. J. Eur. Ceram. Soc. 2016, 36, 2937-2945. [CrossRef]

35. Lame, O.; Bouvard, D.; Wiedemann, H. Anisotropic shrinkage and gravity induced creep during sintering of steel powder compacts. Powder Metall. 2002, 45, 181-185. [CrossRef]

36. Sato, K.; Yilmaz, H.; Hotta, Y.; Ijuin, A.; Watari, K. Dispersion of Ceramic Particles in Aqueous Media with Surface-Grafted Dispersant. J. Am. Ceram. Soc. 2009, 92, 256-259. [CrossRef]

37. Hu, C.; Chen, Y.; Yang, T.; Liu, H.; Huang, X.; Huo, Y.; Jia, Z.; Wang, H.; Hu, L.; Sun, H. Effect of SiC powder on the properties of SiC slurry for stereolithography. Ceram. Int. 2021, 47, 12442-12449. [CrossRef]

38. Manière, C.; Kerbart, G.; Harnois, C.; Marinel, S. Modeling sintering anisotropy in ceramic stereolithography of silica. Acta Mater. 2020, 182, 163-171. [CrossRef] 